\title{
The Canadian Radiosurgical Society Meeting
}

\section{ABSTRACTS}

\author{
Banff, Alberta \\ March 4-5, 2005
}

Can. J. Neurol. Sci. 2005; 32:378-389

Over two beautiful sunny days in Banff, framed by the majestic Rocky Mountains, the Canadian stereotactic radiosurgical community met for the first time. All radiosurgery programs from across Canada participated and over 50 registrants, from all specialties, were represented including neurosurgery, radiation oncology, medical physics, and radiation technology. The objectives of the meeting were to (i) determine if there was sufficient interest in forming a radiosurgical society, (ii) discuss present indications, protocols, and challenges in the field, and (iii) plan multi-centre collaborative trials of stereotactic radiosurgery in Canada. The meeting was a resounding success with all three objectives achieved.

Thanks to sponsorship from four of the six major manufacturers of stereotactic radiosurgery equipment, the conference organizers were able to invite three distinguished keynote speakers. Dr. Michael Schwartz, a neurosurgical pioneer of radiosurgery from Toronto, started off the program by presenting the history of stereotactic radiosurgery in Canada from the first modified linear acceleratorbased program at McGill in 1985, through to the TorontoSunnybrook, Ottawa, Vancouver, Toronto-PMH, Calgary, and Halifax programs, followed most recently by the Gamma Knife ${ }^{\mathrm{TM}}$ units in Winnipeg, Sherbrooke and, imminently, the Toronto Western Hospital. Dr. David Larson, a radiation oncologist from the University of California San Francisco, gave an excellent presentation on the controversies that exist in the field and dissected the dogma to reveal that there is much we do not know and can be learned with the appropriate clinical trials. Dr. Samuel Ryu, a radiation oncologist from Henry Ford Hospital in Detroit, Michigan, discussed his cutting-edge work on stereotactic radiosurgery for spinal disorders, including both metastatic and benign disease. The impressive results of his phase II dose escalation trial for spinal radiosurgery portend an exciting future for the field. All three keynote speakers gave outstanding talks and each was awarded a white cowboy hat, Calgary's symbol of hospitality and respect.

Thirty-three abstracts were presented and are included in these proceedings. The themes were diverse and the presentations were made by radiation oncologists, neurosurgeons, physicists, and radiation technologists. Not only was there much discussion after each talk, but a final round-table session at the conclusion of the meeting lasted 90 minutes (despite the attraction of great skiing).

A major objective, and success, of the meeting was the interest in forming the Canadian Radiosurgery Society (CaRS) that, at least in the near future, will focus on neurologic disease. A formal societal structure was not finalized. However, a multidisciplinary committee comprised of Drs. Michael Schwartz (Toronto), Michael McKenzie
(Vancouver) and James Robar (Halifax) was struck to organize next year's meeting in the Toronto area. The principle of having the organizing committee always include a medical physicist, radiation oncologist, and neurosurgeon was agreed upon, so as to reflect the multidisciplinary nature and practice of this group.

Key goals of CaRS were identified: to educate physicians, to establish guidelines for referral, and to facilitate Canada-wide multicentre studies. The meeting saw the beginnings of just such a collaboration, with Drs. Brian Toyota from Vancouver and Cynthia Ménard from Toronto-PMH presenting results of their own surveys of stereotactic radiosurgery across Canada from both the neurosurgical and radiation oncology perspectives.

Perhaps contrary to the situation south of the border, Canada is well positioned to organize national radiosurgical clinical trials and collaborative outcome analyses. Several conditions were identified that could benefit from standardized treatment and multi-centre outcome data collection. Dr. Ian Parney (Calgary) will spearhead an initiative on brain metastases and the role of stereotactic radiosurgery vs. surgical resection. Drs. Ian Fleetwood (Halifax) and John Wong (Calgary) will focus on vascular malformations, especially inoperable cavernous malformations and those large arteriovenous malformations not amenable to "traditional" radiosurgical techniques. Dr. Elizabeth Yan (Calgary) will tackle pituitary and parasellar benign tumors, and Dr. Brian Toyota, acoustic neuromas. Dr. Normand Laperriere (Toronto) will focus on meningioma treatment, and Dr. Michael West (Winnipeg) will spearhead the functional stereotactic radiosurgery theme. Drs. Brenda Clark (Vancouver) and Chris Newcomb (Calgary) will address the medical physics components of these studies, such as dose specification, quality assurance, and treatment verification. Both radiation technologists and nurses will collaborate to develop and share patient educational materials about radiosurgery.

Despite 20 years of radiosurgery in Canada, only recently has a critical mass of programs formed and matured. Whereas previous regional rivalries and debates about technology threatened to distract those involved in radiosurgery from the real goal of patient care, we have now finally come together with an ambitious plan for crosscountry collaboration. As Dr. Larson concluded, "Everyone was enthusiastic, polite, interested, engaged, and democratic. The meeting was a great success."

It took a long time coming, but we're off to a terrific start. 


\section{BC Cancer Agency Stereotactic Program: Our role in the team}

\section{Christine Alexander RT, Lorraine Geddes RT}

BC Cancer Agency, Vancouver, BC

B.C Cancer Agency has been involved in treating patients with stereotactic radiotherapy and radiosurgery at its Vancouver centre since 1997. During this time we have implemented and refined our treatment process and techniques.

A multidisciplinary approach is adopted throughout the entire treatment process and we will primarily focus on the role of the radiation therapists. The information presented will describe the patients' experience from their initial consultation (New Patient visit) through post treatment care and follow up.

The patient group served by each treatment modality will be identified and the differences in the steps involved in the treatment planning processes with respect to the impact to the patient will be outlined. The treatment day from the perspective of the patient and the individual members of the multidisciplinary team will be shared.

The educational and support resources available to the patient have evolved along with the program. The need to optimise patients' and their family's awareness of these services will be discussed.

\section{Radiotherapy for the Management of unresectable/ recurrent benign and malignant Meningiomas}

Al-Ghamdi, S., Malone, S., Szanto J., Victor, G.

University of Ottawa, Ottawa, Ontario

Purpose/Objective: We analyzed the tumour control and toxicity obtained with radiotherapy in the treatment of meningiomas.

Material and Methods: All patients with intra-cranial meningioma treated at ORCC with radiotherapy (XRT) between 1987-2003 were included. Treatment was as follows: Conventional (C) external beam radiotherapy (EBRT) - 38 patients (29 with surgery), 50-75 Gy (mean: 58 Gy), 1987-2003; 3D conformal EBRT - 22 patients (11 with surgery), 50-60 Gy, 1995-2003; fractionated stereotactic radiotherapy (SRT) - 16 patients, 50-50.4 Gy, 19972001. MRI CT image fusion was used in patients treated with 3DCRT or SRT. SRT was performed using a relocatable frame and bite block. Planning target volume included a margin of $2-5 \mathrm{~mm}$ around gross tumour volume for benign and 10-20 $\mathrm{mm}$ for malignant tumours. SRT was planned using the X-Knife treatment planning system.

Results: At the time of radiotherapy, 31 (41\%) had benign and 19 (25\%) had malignant tumours, 22 (29\%) had unknown differentiation level (12 had previously documented benign pathology). Number of prior surgeries was: none (46\%), 1 (68\%), 2 $(22 \%),>2$ surgery $(10 \%)$. Seventy percent of tumours were in the skull base. Mean XRT dose was 53-54 Gy for benign or unknown and 60 Gy for malignant tumors. Amongst those receiving combination therapy, surgery was performed prior to radiotherapy. Fifty-six percent of malignant tumours were completely resected. $2 / 5$ year progression free survival was $93 \% / 88 \%$ for all patients (97\%/89\% for benign or unknown differentiation and $86 \% / 86 \%$ for malignant tumours). Toxicity potentially related to radiotherapy in patients treated with CEBRT/3DCRT was (\#pts/\#pts): overall (13/3), stroke (2/1), neurocognitive deficits $(9 / 2)$, unstable gait $(1 / 0)$, tinnitus (1/0). There was no recorded toxicity for SRT.
Conclusion: Radiotherapy is effective management of unresectable or recurrent meningiomas of benign and malignant histology. With modern 3DCRT and SRT techniques the risk of serious morbidity is low.

\section{Embolization prior to radiosurgery reduces the obliteration rate for AVMs.}

Yuri M. Andrade-Souza, Meera Ramani, Daryl Scora, May N. Tsao, Karel terBrugge, Michael L. Schwartz

Division of Neurosurgery and Department of Radiation Oncology, Sunnybrook and Women's College Health Sciences Centre; University of Toronto, Ontario, Canada.

Objective: To analyze the impact of liquid embolization (enbucrilate) prior to radiosurgical treatment in the obliteration rate for brain AVMs.

Method: Thirty-nine patients with embolization prior to radiosurgical treatment were matched according to marginal dose, volume and anatomical location with 39 other patients (control) selected randomly that did not have prior embolization.

Results: The median follow-up was 42 months (range 27-118). The median age of the patients was 37 years (range 5-67, mean 35.9) and $51.3 \%$ were women and $48.7 \%$ were men. There was no difference in relation to volume (mean: $6.45 \mathrm{~cm}^{3}$ ), marginal dose (mean: 17.3Gy) or AVM location between both groups. The mean radiosurgical-based AVM score and the OPI were $1.56(\mathrm{SD}=0.52)$ and 11.6 (SD: 5.2) for the group that had prior embolization and 1.62 $(\mathrm{SD}=0.54)$ and 11.4 (SD: 4.2) for the control group (T-test, $\mathrm{P}=0.61$ and $\mathrm{P}=0.83$, respectively). The obliteration rate was $53.8 \%$ for patients with prior embolization compared to $79.5 \%$ for the control group (Pearson Chi-square, $\mathrm{P}=0.016$ ).

Conclusion: Embolization prior to radiosurgery decreases the obliteration rate for equivalent AVMs (same volume, marginal dose and location).

\section{Linac radiosurgery for treatment of severe epilepsy related to hypothalamic hamartoma}

\section{JM Caudrelierl ${ }^{2}$, D Keene ${ }^{2}$, J Ventureyra $^{3}$, T Nguyen $^{4}$, J Michaud $^{5}$}

${ }^{1}$ Department of Radio Oncology, Ottawa Hospital Regional Cancer Centre, Ottawa

${ }^{2}$ Department of Neuropediatry, Children Hospital Eastern Ontario, Ottawa

${ }^{3}$ Department of Neurosurgery, Children Hospital Eastern Ontario, Ottawa

${ }^{4}$ Department of Neuroradiology, Ottawa Hospital, Ottawa

${ }^{5}$ Department of Pathology, Children Hospital Eastern Ontario, Ottawa

Introduction: We report and discuss irradiation treatment as linac radiosurgery indicated for severe and intractable epilepsy related to hypothalamic hamartomas (HHs) in two young boys. HHs are uncommon disease and patients frequently present gelastic seizures which are refractory to drug therapy. During evolution of the disease, other types of intractable seizures occur, often with falls and in addition precocious puberty and progressive mental deterioration. The evolution of the disease is considered to be invariably unfavourable. Role of $\mathrm{HHs}$ in genesis of seizures has been demonstrated by stereo-EEG recording. In this area, neurosurgical resection of $\mathrm{HHs}$ is associated with a very high risk of complications including vascular events and hemiparesis. Improvements of symptoms have been reported in small series of patients treated by 
gamma-knife radiosurgery (as in a combined analysis of ten worldwide cases).

Material and results: Two young boys, nine and 12 years old diagnosed with HHs following onset of seizures during infancy have been previously treated by neurosurgery. Both experienced complications with vascular events and hemiparesis. Diagnostic was proven from surgical resection. They have recovered over time from hemiparesis but have experienced different type of seizures refractory to medical treatment and progressive mental retardation and precocious puberty. On MRI done over years, there was no growth of tumor. After a multidisciplinary consult, a single irradiation as linac radiosurgery was proposed. A single treatment is the most appropriate treatment as the patients are non-cooperative and tests such as MRI or CT-scanner or irradiation delivery have to be performed under general anesthesia. MRI with thin slices (2-3 $\mathrm{mm}$ ) and fluid attenuated inversion recovery (FLAIR) sequences was done to delineate $\mathrm{HHs}$ and sensitive structure, mainly chiasma and optic bands. A single isocentre and a circular collimator were used to deliver a prescribed dose of $15 \mathrm{~Gy}$ inside the periphery of the lesion. The prescribed dose was also chosen to limit the chiasma or optic bands dose at 10 Gy maximum. In the same time, this treatment is functional and not tumoral. Therefore, there is probably no need to cover all the target volume as done for benign or malignant tumor control. The two treatment procedures were eventfree. One boy has had a follow-up of 19 months and has experienced improvement of seizure. The second one was treated recently and more follow-up is needed for assessment of results.

Conclusion: HHs are a very uncommon disease with children suffering from severe epilepsy refractory to medications. The life is usually miserable for patients and family. Few data are available for HHs treated by radiosurgery. These two boys could be the first patients treated in Canada by Linac radiosurgery. HHs are cases for functional stereotactic irradiation where the goal of treatment differ from usual tumoral targets.

\section{Stereotactic Fractionated Radiotherapy for the Treatment of Benign Meningiomas - the British Columbia Experience}

Charles Candish ${ }^{l}$, Brenda Clark ${ }^{2}$, Roy Ma ${ }^{l}$, M. Martin ${ }^{3}$, Michael McKenzie ${ }^{l}$

Departments of Radiation Oncology ${ }^{1}$, Medical Physics ${ }^{2}$, and Radiology ${ }^{3}$, BC Cancer Agency, Vancouver, British Columbia, Canada.

Purpose: To assess the feasibility, efficacy and toxicity of stereotactic fractionated radiotherapy (SRT) in the treatment of meningiomas.

Methods: A retrospective review of all cases of meningioma treated at the BC Cancer Agency (Vancouver Centre) was undertaken.

Results: Between April 1999 and October 2004, 38 patients underwent SFT. Of 34 patients (36 tumors) assessed, the median age was 53 years. The indication was primary treatment in 26 patients (no histology). Four received immediate post-operative treatment and six received treatment on progression. Commonest sites were cavernous sinus (17), optic nerve (6), and cerebellopontine angle (5). The mean clinical target volume and planning target volume were $11.9 \mathrm{~cm}^{3}$ (range, $0.6-38.4 \mathrm{~cm}^{3}$ ) and $18.9 \mathrm{~cm}^{3}$ (range, $2.0-46.5 \mathrm{~cm}^{3}$ ) respectively. Treatment was planned using the BrainLAB system and delivered with 6MV photons with static conformal fields (custom made blocks, nine patients and micro-multileaf collimator, 25 patients). Median number of fields was 6 (range, 4 to 10). The median dose prescribed was $50 \mathrm{~Gy}$ (range $45 \mathrm{~Gy}$ to $50.4 \mathrm{~Gy}$ ) in 28 fractions (range, 25 to 29). The mean homogeneity and conformality indices were 1.1 and 1.9 respectively. Treatment was well-tolerated. Median follow up was 26 months with no evidence of recurrence in any patient as yet. One patient developed an area of possible radionecrosis related to previous radiotherapy, and two men developed mild hypogonadism necessitating testosterone replacement. The vision of all patients with optic pathway meningiomas improved or remained static.

Conclusion: SRT for the treatment of meningiomas is practical, safe and with early follow-up, seems to be effective.

\section{Stereotactic Radiosurgery for Brain Metastases: The Calgary Tom Baker Cancer Centre Experience}

A Chan, D Spencer, E Yan, I Parney, C Newcomb, $P$ Craighead, $Z$ Kiss

Alberta Radiosurgery Centre, Calgary, Alberta, Canada

Whereas stereotactic radiosurgery (SRS) benefits cancer patients with $<3$ brain metastases, good performance status and controlled primary diseases, it is likely underutilized in Canada. Our aim with this report is to audit our results and median survival of patients with brain metastases treated with SRS between April 2000 and May 2004.

The Tom Baker Cancer Centre at the University of Calgary treated 41 such patients ( 19 males and 21 females) with SRS. The median age was 58 years (range: $28-84$ years) and primary tumor sites included lung (12), breast (9), colorectum (3), melanoma (7), kidney (7), esophagus (1), prostate (1) and one patient with an unknown primary. Median interval between their initial diagnosis and their brain metastases was 15.8 months (range: 0-160 months). Using the Radiation Therapy and Oncology Group RPA classification, 12 patients were class I, 25 patients were class II and 4 patients were class III.

Treatment was delivered using the Radionics X-Knife system with $6 \mathrm{MV}$ photon and circular collimators. The median prescribed marginal dose was 18 Gy (11 to $30 \mathrm{~Gy}$ ), usually prescribed to the $80 \%$ isodose volume. The median collimator size used was $2.0 \mathrm{~cm}$ (from 0.75 to $4.0 \mathrm{~cm}$ ). Twenty-one of the 31 patients who received SRS as the primary therapy also had whole brain radiation.

Median survival was 10.2 months: $13.5,6.5$ and 1.8 months for RPA class I, II and III, respectively. The median survival for patients who did not have extracranial metastases was 11.9 months, compared to 6.7 months for those who had extracranial metastases. As of December 2004, 13 patients are alive and 28 patients had died. 21 of which died of progression of extracranial metastases. Thirty patients had no CNS disease progression at their last censor visit or at the time of death. We have 12 patients who have survived beyond 16 months after the diagnosis of the brain metastases, most of whom were in the RPA class I or II with no extracranial distant metastases.

Stereotactic radiosurgery can provide good symptomatic palliation for patients with brain metastases. For patients without extracranial metastases, it may even provide survival benefit in selected patients. 
7. Audiologic and Treatment Outcomes Following LINACbased Stereotactic Irradiation for Acoustic Neuroma

Chung HT, Ma R, Toyota B, Lee AS, Clark B,

Robar J, McKenzie $M$

Radiation Oncology, BC Cancer Agency and Neurosurgery, Vancouver General Hospital, Vancouver, BC.

Purpose: Although surgical excision remains the gold standard for treatment of acoustic neuroma, radiotherapy is gaining momentum as an alternative. This is particularly evident in patients with useful hearing, where fractionated radiotherapy offers the potential for hearing preservation. Our objective was to determine disease control, hearing preservation (via audiograms) and toxicity following LINAC-based stereotactic radiation for acoustic neuroma.

Methods: Seventy-two acoustic neuroma patients underwent stereotactic irradiation, and had $\geq 1$ follow-up from October 1997 through March 2002. Forty-five received single-fraction stereotactic radiosurgery (SRS) and 27 received fractionated stereotactic radiotherapy (SRT). Prior to treatment, all SRS patients were functionally deaf while 23/25 SRT patients had useful hearing in the affected ear. The minimum peripheral dose was 12Gy and 45Gy in all SRS and SRT patients, respectively. Tumour control, toxicity and hearing preservation was recorded.

Results: Median follow-up in the SRS and SRT groups were 27 and 26 months, respectively. No progression of the tumour has been seen post-SRS and SRT. Based on audiogram criteria, the 1- and 2year hearing preservation rates were $85 \%$ and $57 \%$, respectively. The mean pre- and post-SRT speech recognition threshold $(\mathrm{dB})$ was 20 and 38, respectively. The mean pre- and post-SRT speech discrimination (\%) was 91 and 59, respectively.

Conclusions: Stereotactic irradiation achieves good local control, with acceptable toxicity. Fractionation of radiotherapy appears to provide improved rates of hearing preservation.

\section{Does Intensity Modulation Improve Healthy Tissue Sparing in Stereotactic Radiosurgery of Arteriovenous Malformation?}

BG Clark' ${ }^{l}$; M McKenzie ${ }^{l}$; JL Robar ${ }^{l}$; E Vollans ${ }^{l}$; B Toyota ${ }^{2}$; A Lee ${ }^{l}$; $R \mathrm{Ma}^{l}$; K Goddard , S Erridge $^{l}$.

${ }^{1}$ Radiation Oncology, BC Cancer Agency and ${ }^{2}$ Neurosurgery, Vancouver General Hospital, Vancouver, BC.

The aim of this study is to investigate conformal stereotactic radiosurgery (SRS) techniques and quantify potential advantages of intensity modulation for stereotactic radiosurgery of arteriovenous malformation (AVM).

Twenty patients treated with SRS for AVM between 1998 and 2001 were replanned with each of four techniques: circular noncoplanar arcs, dynamic arcs, static conformal fields and intensity modulated fields (IMRS). Patients were selected for size and irregular target shape; with a maximum AVM dimension at least $20 \mathrm{~mm}$ or volume greater than $10 \mathrm{~cm}^{3}$. Target volumes ranged from $2.12 \mathrm{~cm}^{3}$ to $13.87 \mathrm{~cm}^{3}$ with a median of $6.03 \mathrm{~cm}^{3}$. Resulting dose distributions were analysed to determine conformality to AVM, target dose homogeneity and healthy tissue dose at 3, 6, 12, 18 and 24 Gy.

The results show an improvement in conformality index with differences statistically significant $(\mathrm{p} \leq 0.01)$ between circular arcs and either conformal or IMRS, conformal and dynamic arcs and IMRS and conformal. The paired differences between dose homogeneity index are significant for improvement in moving from circular arcs to either conformal or IMRS, conformal to IMRS ( $\mathrm{p} \leq$ 0.001 ) with no difference between conformal and dynamic arcs.

The normal tissue analysis data shows a substantial reduction in dose at $24 \mathrm{~Gy}$ for all other techniques and an increase in dose at three Gy for the static field techniques when compared to the arc techniques. Overall, no advantage was seen for intensity modulation and the dynamic arc technique offers the greatest potential for minimization of normal tissue dose at all dose levels.

\section{Establishing a dedicated Radiosurgery program within a publicly funded health care system: A realistic goal?}

Craighead PS (lead) ${ }^{*, 3}$, Chan $A^{*, 3}$, Kiss $Z^{2,3}$, Parney $I^{2,3}$, Yan $E^{*, 3}$, Newcomb $C N^{*, 3}$, Spencer $D^{*, 3}$, Merritt $S^{*}$, Inkson $T^{2}$.

* Departments of Radiation Oncology and Medical Physics, Tom

Baker Cancer Centre, Calgary, Alberta.

${ }^{2}$ Department of Clinical Neurosciences, Calgary Health Region, Calgary

${ }^{3}$ University of Calgary

Introduction: The majority of provinces within Canada have separate funding agencies for cancer and neurosurgery disciplines, resulting in a schism in how to advance academic specialty areas such as radiosurgery. The Tom Baker Cancer Centre quickly realized this conundrum when it provided once weekly treatment capacity on a single linear accelerator in 1999; it had neither the funding, nor the organizational support, to use this technology for a full spectrum of conditions.

Method: In 2002 an informal working group met to devise a strategy of how to advance radiosurgery in Alberta. Attendees came from radiation oncology, neurosurgery, medical physics, neurooncology, and administration. This group strongly endorsed the development of a dedicated program for radiosurgery. A demographic search was performed to analyse the true need for such technology in Calgary. Once completed, a strategic plan was developed that was passed onto the executive bodies of the Calgary Health Region, and Tom Baker Cancer Centre. This plan discussed the unique collaboration that would come from this program, the objectives for establishing it, and the steps needed for this to become a reality.

Results: In this presentation we will show the critical elements that need to be addressed for a collaborative radiosurgery program such as the Alberta Radiosurgery Centre in Calgary. The presentation will show how these issues were faced in Calgary, and important lessons from our experience. Some of these include details around staffing, budgets, development of rounds, attainment of equipment, and governance. The Alberta Radiosurgery Centre opened its doors to patient care in November 2004. A program manager has appointed staff, who have received training, and now operate from within the centre. This manager reports to a conjoint committee representative of the health region and cancer centre. Academic and research issues are the purview of a multidisciplinary advisory group within the program. The centre currently operates on a daily basis, with single fraction treatments constituting a full day during the week, and fractionated treatments currently using two thirds of the capacity. The centre has an established referral mechanism that targets access for consultation within two 
weeks. Brain metastases patients are consulted and treated within a maximum of two weeks.

Conclusion: The development of this centre has focused resources, developed expertise, and significantly increased access to patient care. Our future goals are to develop research opportunities, increase collaboration, and enhance the careers of medical and paramedical specialists in radiosurgery. If we can do this while retaining access to high quality patient care, this will confirm the benefit of developing a dedicated program for such patients.

\section{Stereotactic Radiation Therapy Program at Princess Margaret Hospital}

Heydarian M, Michaels H, McKinnon S, Ladak S, Millar BA, Menard $C$, and Laperriere $N$

Princess Margaret Hospital, University Health Network, University of Toronto, Toronto, Ontario, Canada

Radionics treatment planning and delivery systems and a dedicated Clinac 2100C/D are used at our institution for stereotactic radiosurgery/radiotherapy (SRS/SRT) of intracranial lesions. Immobilisation of patients is based on commercially available CRW (for SRS), Gill-Thomas-Cossman (GTC), Head and Neck (HNL) and paediatric (TLC) relocatable frames. Different techniques, including non-coplanar arc therapy, step and shoot 3D conformal and intensity modulated stereotactic radiotherapy (IMSRT) are used for treatment of CNS, ENT, Endocrine, Eye and Paediatric cases (overall 782 to date). Circular collimators of 10 to $50 \mathrm{~mm}$ diameter with $2.5 \mathrm{~mm}$ increments are used for the arc technique for treatment of small lesions. A Mini Multileaf Collimator (MMLC) of $4 \mathrm{~mm}$ leaf thickness and 10x12 cm maximum field size at the isocentre is also used for stereotactic 3D conformal and IMSRT techniques for treatment of larger lesions. Different techniques will be compared with each other and sample cases of each technique will be presented. Also, comparisons are made with a Varian 2100EX and an Elekta SL25, which are now available to our stereotactic clinic as well as with a dedicated Elekta "Synergy S" machine with the KVI Cone-Beam CT feature, which becomes available to our stereotactic clinic by end of March 2005. Other systems such as the Gamma Knife and PET, which are being added to our stereotactic program in 2005, will also be addressed.

\section{Comparison of Different MLC Systems for IMSRT of Ethmoid Tumours}

Heydarian M, Sadrolhefazi B, Fung B, Ringash J and Cummings B

Princess Margaret Hospital, University Health Network, University of Toronto, Toronto, Ontario, Canada

Radionics treatment planning and delivery systems, including a Mini Multileaf Collimator (MMLC) are routinely used at our institution for intensity modulated stereotactic radiotherapy (IMSRT) of intracranial lesions. The MMLC has a leaf thickness of $4 \mathrm{~mm}$ and maximum field size of 10x12 cm at the isocentre. A Varian 2100EX (of $5 \mathrm{~mm}$ leaf thickness at the centre) has also been commissioned for the above techniques to eliminate the maximum field size limitation of Radionics MMLC. Dosimetric comparisons are made for treatment of two Ethmoid tumours of medium (37 cc) and large $(115 \mathrm{cc})$ sizes, which both involve organs at risk (OAR) such as Optic Chiasm and Nerves, and the Brainstem. The same beam setup and optimisation parameters were used for both MLC systems. Parameters used for the comparison include: $2 \mathrm{D}$ and $3 \mathrm{D}$ dose distributions, dose heterogeneity (DH), maximum, minimum and median doses as well as dose volume histograms (DVH) for the target volume; and maximum dose, median dose, dose of $10 \%, 20 \%$ and 50\% volumes (D10, D20 and D50) and DVHs for OARs. The OARs dose volume data are presented for a normalised dose, when $95 \%$ of the tumour received at least $95 \%$ of the prescribed dose. DVH comparisons have shown that the two systems are overall clinically comparable. However, the Radionics MMLC had the advantage of delivering the prescribed doses using $9 \%$ fewer segments and $35 \%$ less number of monitor units for the above cases and therefore less dose to the surrounding normal structures by up to $7 \%$.

\section{Application of Routine RTOG QA Measures in Stereotactic Radiosurgery}

Fred Hsu, Dr. Liam Mulroy, James Robar, Dorianne Rheaume, Ian Fleetwood, Mammo Yewondwossen, Jason Schella

Radiation Oncology Medical Physics and Neurosurgery, Dalhousie University, Halifax

Quality assurance guidelines have been developed by the Radiation Therapy Oncology Group (RTOG) for stereotactic radiation. These are useful in assessing compliance with dosimetry requirements in clinical trials and in daily radiotherapy practice.

The RTOG guidelines define dose homogeneity within the target volume as the ratio of the maximum dose to the prescription dose (MD/PD ratio). This is known as the homogeneity index and is considered optimal when less than or equal to 2 . The conformity of the prescription dose to the target is defined by the ratio of the prescription isodose surface volume to the target volume (PIV/TV ratio). Values between 1 and 2 for this ratio are optimal.

Data regarding the above measures were collected for 31 patients treated with SRS using the BrainLAB stereotactic planning system between Dec 2002 and Dec 2004 at the Nova Scotia Cancer Centre; three patients had multiple lesions treated. There were 22 patients with AVMs, seven with metastases, one with an acoustic neuroma and one with a pituitary adenoma. Preliminary analysis shows minimal variation in the homogeneity index (MD/PD) with a range of values from 1.26 to 1.53 . However, there is substantial variation in the conformity index (PIV/TV) with values ranging from 1.29 to 6.96 and $10 / 37$ target lesions with values above 2 . Further analysis of the data will be carried out to assess the impact of such factors as small versus large target volume and multiple targets treated in the same patient.

\section{Gamma Knife radiosurgery for trigeminal neuralgia; preliminary results from the Québec Gamma Knife at the Université de Sherbrooke Hospital}

Brendan G Kenny MD, David Mathieu MD, Rachel Bujold MD, André Bertrand, Caroline Bazinet and Josée Dumont .

Department of Neurosurgery and Radiation Oncology, CHUS, Fleurimont, Québec, Canada

The Québec Gamma Knife was inaugurated and the first patients treated in August 2004. To date more than 100 patients with a variety of diagnoses including metastases, acoustic tumours, meningiomas, 
pituitary tumours, arteriovenous malformations, cavernomas and trigeminal neuralgia have been treated. Thirty-two patients have been treated for Trigeminal Neuralgia of whom 17 have been followed for two months or more. Patients were treated on an outpatient basis with a single dose of 80 Gy to the pre-pontine portion of the affected nerve using the $4 \mathrm{~mm}$ collimator following MRI targeting. There were 12 males and five females aged 50-86 (median 72). Four patients had recurrent pain following previous surgery,

Results: Ten patients are pain free; four without medication, four with reduced and two with the same medication. Seven patients have reduced severity/frequency of pain to $10-50 \%$ pre-radiosurgery with the same or reduced medication. All patients judged their result either excellent or good. Other than one patient with minor facial tingling no side effects have been reported.

Conclusions: Our early experiences confirms that Gamma Knife radiosurgery for trigeminal neuralgia is very well tolerated by all patients including the elderly, and that results are very promising with no immediate or short-term complications.

\section{Princess Margaret Hospital experience with fractionated stereotactic radiotherapy for acoustic neuroma}

Koh ES, Millar BA, Ménard C, Michaels H, Heydarian M, Ladak $S$, McKinnon S, Laperriere NJ.

Department of Radiation Oncology and Clinical Physics, Princess Margaret Hospital,University of Toronto, Toronto, Ontario.

We report our experience in our first 66 consecutive patients treated with stereotactic radiation therapy (SRT) for acoustic neuroma from October 1996 to February 2005. Five patients were excluded, two NF-2 associated, two discontinued at 28 Gy and 30 $\mathrm{Gy}$, and one was treated with single fraction radiosurgery (15.5 Gy to the $90 \%$ isodose).

Sixty-one patients were analyzed, 32 males and 29 females, age range $18-80$ years (median 58). Median primary tumor volume was $4.9 \mathrm{cc}(0.3-49)$. Imaging progression occurred in 28 (45.9\%) and symptom progression in eight $(13.1 \%)$. Presenting symptoms included tinnitus $(52.5 \%)$, gait instability (49.2\%), CN V numbness $(32.8 \%)$, facial nerve weakness $(13.1 \%)$, and trigeminal neuralgia (4.9\%). 58/61 (95.1\%) had some degree of hearing loss and 24/61 $(39.3 \%)$ had useful hearing at baseline. Formal audiology at baseline was documented in $76 \%$.

Sixty patients received 50 Gy in 25 fractions, one received 52 Gy. Acute toxicities included grade I fatigue and nausea in $43 \%$ and $41 \%$ respectively, grade I headache $(20 \%)$, occasional vomiting (5\%). Grade II toxicities occurred in 5\%. Most pre-existing cranial nerve V and VII dysfunction remained stable. No new cranial nerve palsies developed. One case of RT induced glioblastoma occurred 5.8 years post therapy.

At a median follow up of 23.4 months, actuarial progression free survival is $98 \%$. One patient experienced tumour progression at 2.3 months post RT and underwent resection. Hearing function remained stable in $77 \%$ for all patients, in $82 \%$ and $67 \%$ with baseline useful and non useful hearing. Six percent noted improvement if initial hearing was useful.

Conclusions: FSRT prescribed to $50 \mathrm{~Gy}$ in 25 fractions is well tolerated with an excellent crude local control rate of $98.3 \%$ comparable to published literature.

\section{Brain Radiosurgery: Foundations, Methods, Results and Questions}

David A. Larson, PhD, MD

Department of Radiation Oncology,

University of California San Francisco

Although radiosurgery (RS) developed empirically, its formal foundations are based on the results of Phase I-III trials for brain metastases or malignant gliomas. These trials demonstrate both a general dose-volume relationship and, specifically for brain metastases, that RS for some subsets of patients may result in decreased steroid requirements and modestly increased survival. These trials, and the retrospective series which motivated them, are less clear about important issues such as dose response for efficacy, target margin, response for different target enhancement patterns, appropriate number of metastatic tumors to treat with RS effects of whole brain RS dose for large number of targets, whether WBRT should be deferred until possible post-RS brain progression, and degree of dose conformity required. For malignant glioma patients some issues are similar, though they seem less pressing given that Phase III data indicate that RS may add no benefit when delivered preceding XRT/BCNU. Retrospective data for benign brain tumors such as meningioma and acoustic neuromas generally indicate that RS and fractionated stereotactic radiosurgery (FSRT) outcomes are similar, even though FSRT patients are from a less restricted subset of presentations. Long-term risks of RS include cyst formation or secondary tumor induction. Claims that any particular RS technique is best tend to be balanced by counterclaims favouring another technique, and most clinical data do not support the notion that any particular methodology is clinically superior. Numerous clinical questions remain, some of which may yet be addressed through formal trials.

\section{Three dimensional radiosurgery dose verification using polymer gel dosimeters}

A. Bertrand ${ }^{l}$, G. Leclerc ${ }^{l}$, B. Kenny ${ }^{l}$, M. Lepage Le $^{2}$

${ }^{1}$ Département de radio-oncologie

${ }^{2}$ Centre d'Imagerie Métabolique et Fonctionnelle

Centre Hospitalier Universitaire de Sherbrooke and Université de Sherbrooke

Introduction: Polymer gel dosimetry has the potential for three dimensional dose verification with sub-millimetric spatial resolution. Upon irradiation, a polymerization reaction involving acrylamide (AA) and N,N'-methylene-bisacrylamide (BIS) generates changes in the optical properties of the irradiated region. In addition, the transverse magnetic relaxation time (T2) decreases as a function of the absorbed dose and magnetic resonance imaging (MRI) can be used to record the absorbed dose distribution. This novel type of dosimetry is extremely well suited for the experimental verification of complicated treatment plans such as those required for stereotactic radio-surgery (SRS) and IMRT treatments.

Materials and methods: Tissue-equivalent normoxic polymer gel dosimeters were fabricated from $4.5 \%$ AA, $4.5 \%$ BIS, $5 \%$ gelatin and $86 \%$ water, all by weight. Ten $\mathrm{mM}$ of tetrakis(hydroxymethyl)phosphonium chloride was added to the solution before gelation. Gels were subjected to a SRS treatment using a Leksell Gamma Knife and planned using the Leksell Gamma Plan. The absorbed dose distribution was determined using a multi- 
echo spin echo sequence with either a $1.5 \mathrm{~T}$ or a $7 \mathrm{~T}$ MRI scanner.

Results and discussion: The absorbed dose from various SRS irradiations was determined with polymer gel dosimeters. Good agreement between the expected beam location and size for different collimator sizes was obtained. A similar good agreement was found for a treatment with sharp spatial variations at the limit of the gamma-knife possibilities.

Conclusions: High-resolution polymer gel dosimetry is a valuable tool in SRS treatment planning verification.

\section{Updated experience in stereotactic radiosurgery for arteriovenous malformations at the British Columbia Cancer Agency and Vancouver General Hospital}

McKenzie, Michael ${ }^{1,3}$; Toyota, Brian'2, 3; Clark, Brenda Gillian ${ }^{1,3}$; Lee, Andrew ${ }^{l}$; Vollans, Emily ; Robar, James Leonard ${ }^{l}$; Ma, Roy ${ }^{1,3}$; Goddard, Karen ${ }^{1,3}$

${ }^{1}$ Radiation Oncology, BC Cancer Agency, Vancouver, BC, Canada.

${ }^{2}$ Neurosurgery, Vancouver General Hospital, Vancouver, BC,

Canada.

${ }^{3}$ University of British Columbia, Vancouver, BC, Canada.

Purpose/Objective: To review local experience with linearaccelerator based stereotactic radiosurgery (SRS) for arteriovenous malformation (AVM).

Design: Single-arm prospective cohort.

Materials/Methods: Patients were selected for SRS if deemed inoperable. All patients were followed prospectively. All patients were encouraged to undergo angiography at three years post-SRS or when MRI suggested possible obliteration.

Results: Between 1997 and 2004, 64 patients were treated. Median age was 43 (range 10-72) years. Previous treatments included prior SRS at another centre in four patients. Median nidus volume was 2.69 (range 0.11-15.9) cc. AVM was centred in the corpus callosum, basal ganglia, or brainstem in 20 patients. Median prescribed (marginal) dose was 20 (range 12-25) Gy. Median potential follow-up was 58 (range 8-87) months. The four year actuarial rate of performance of follow-up angiography was $82.2 \%$. In patients undergoing follow-up angiography and in the cohort as a whole, the 4 year actuarial rates of complete obliteration were $78.4 \%$ and $65.9 \%$, respectively. Two patients were retreated for persistent nidus with SRS 37 and 40 months following initial treatment. In both, complete obliteration was documented subsequently. The one year actuarial rate of transient SRS-related neurological deficit requiring steroids was $22.9 \%$. The four year actuarial rates of any symptomatic radionecrosis and radionecrosis requiring hospitalization were $26.7 \%$ and $4.4 \%$, respectively.

Conclusions: The complete obliteration rate is comparable to previous reports. The rates of SRS-related side effects likely reflect the size and location of the AVM's treated in this cohort, and close follow-up. Analysis of predictors of adverse events will be presented.

\section{Stereotactic Radiotherapy in Canada: A Survey}

Cynthia Ménard, Barbara-Ann Millar, Normand Laperriere, David Jaffray

Princess Margaret Hospital, University of Toronto, Toronto, Ontario

Introduction: Rapidly evolving technologies are enabling highprecision radiotherapy and have led to changes in: 1) patterns of clinical practice 2) equipment for delivery of radiation, and 3) modification of radiation therapy techniques. To enable the development of collaborative work, and optimize use of resources across Canada, it is essential to develop a consistent standard of care and delivery. This would allow valid comparison and pooling of data in the management of disease, and encourage the formation of joint research within this specialty field.

Methods: We plan to conduct a survey of all Canadian radiotherapy centres in order to ascertain and to summarize the current standard of practice and technology. The survey will consist of a technical and a clinical module. Specific questions will address the following themes: clinical utilization, patterns of practice, equipment, imaging resources, treatment planning techniques, dose prescription conventions, personnel, research goals and future directions. The influence of the availability of resources and the impact of this on clinical practice will also be reviewed.

Discussion: The survey information will allow a broad review of current practice, and will serve as a platform upon which judicious national consensus guidelines and nation-wide research programs can be launched.

\section{A comparison of stereotactic radiosurgery systems: a time- motion study}

Newcomb $\mathrm{CH}^{1,2}$, Chan $\mathrm{A}^{1,2}$, Craighead $P S^{1,2}$, Hudson $I^{I}$, Kiss $Z H T^{2,3}$, Merritt $S^{l}$, Parney $I^{2,3}$, Spencer DP ${ }^{1,2}$, Yan $E^{1,2}$

1 Departments of Radiation Oncology and Medical Physics, Tom Baker Cancer Centre, Calgary, Alberta

${ }^{2}$ University of Calgary

${ }^{3}$ Department of Clinical Neuroscience, Calgary Health Region

The purpose of this study is to compare efficiencies prior and following the introduction of a dedicated Novalis Stereotactic system for single fraction radiosurgery and multiple fraction radiotherapy. The study was performed at the Tom Baker Cancer Centre, in Calgary, Alberta, Canada. The study consisted of two measurement periods separated by one year. During each measurement period procedure times, patient and staff involvement were recorded and compared for immobilization, CT simulation, planning and delivery. While results are preliminary, several major changes in the overall process have been possible:

1. Switching from a plaster-of-paris to an aquiplast system has allowed immobilization and CT simulation for fractionated treatments to be performed in a single booking. Better integration of the planning and delivery systems has eliminated the need for an additional conventional simulation. In total, three visits to the clinic by the patient have been eliminated and the time to treat reduced by more than one week.

2. By using dedicated staffing, fractionated plans are now fasttracked through planning, reducing the time to treat by an additional two weeks. 
3. By using a machine with improved isocentre accuracy there is no longer a need to check and realign patient position with each couch rotation. Previously, each field would require at least 20 minutes for alignment and delivery. A complex case could require more than 90 minutes for delivery. The improvement in machine precision has resulted in both more complex treatment optimizations, up to seven conformal arc fields are now standard, being delivered within a 15-20 minute treatment booking. This has had considerable impact on patient comfort and increased the scope of target type and location being treated.

\section{The BC Cancer Agency experience of radiosurgery for brain metastases}

Alan Nichol*, Brian Toyota ${ }^{\dagger}$,Michael McKenzie ${ }^{*}$, Brenda Clark $^{\ddagger}$, Andrew Lee ${ }^{\dagger}$, Roy Ma* Emily Vollans ${ }^{\ddagger}$, Richard Lee ${ }^{\ddagger}$,Ermias Gete

${ }^{*}$ Radiation Oncology, ${ }^{\dagger}$ Neurosurgery, ${ }^{\ddagger}$ Medical Physics, Vancouver Cancer Centre, BC Cancer Agency, Vancouver, BC

Purpose: To analyse prognostic factors and classification schemes for brain metastasis patients treated with stereotactic radiosurgery (SRS).

Materials and Methods: Between June 1997 to Oct 2004, 68 patients with 1-3 brain metastases were approved for treatment by the BC Cancer Agency Stereotactic Conference. Most patients received SRS as a boost after $30 \mathrm{~Gy} / 10$ whole brain radiotherapy (WBRT), but SRS was also used for salvage after craniotomy and/or 20Gy/5 WBRT. The patients had a median age of 55 (35-78) and a median KPS of 80 (40-100). Twenty-five patients had non-small cell lung, 16 had breast and 27 had other cancers. The largest lesion per patient had a median diameter of $20 \mathrm{~mm}$ (3-31) and the lesions were treated to a median dose of 18 Gy (13.5-24) prescribed to the $80 \%$ isodose surface.

Results: The median survival for the cohort was 13.7 months. Univariate analysis of the effects of Age, KPS, Number of Lesions, Volume of Largest Lesion, Control of Primary, Presence of Extracranial Metastases and Primary Malignancy determined that only younger age predicted significantly for better survival $(p=0.04)$. For each patient, the Radiation Therapy Oncology Group recursive partitioning analysis (RTOG RPA) class, score index for radiosurgery in brain metastasis (SIR) and the basic score for brain metastasis (BS-BM) were determined, but they did not predict significantly for survival.

Conclusions: For this cohort of patients selected by the BC Cancer Agency Stereotactic Conference, the median survival compared favourably with other reports. Only younger age predicted for better survival.

\section{A comparison of planning methods for the treatment of acoustic neuromas using linear accelerator based fractionated stereotactic radiotherapy}

Parker W., Roberge D., Patrocinio H., Patel S., Souhami L., Deblois F., Ruo R., Podgorsak E.,

McGill University Health Centre, Montreal, Quebec.

Acoustic neuromas are often treated with stereotactic radiotherapy (SRT) or radiosurgery (SRS). At our institution, SRT is delivered using a tertiary micro-multileaf collimator (mMLC). If these patients could be treated using a dynamic beam delivery inverse planning-IMRT system with a conventional MLC gains could be made in convenience and workflow. Our aim was to compare our mMLC technique to a similar inverse planned standard MLC IMRT technique. A retrospective planning study was undertaken for ten patients, previously treated using mMLC-based SRT delivering $45 \mathrm{~Gy}$ in 20 fractions to the periphery of the PTV using non-coplanar $6 \mathrm{MV}$ photon beams. The patients were replanned with the inverse planning-IMRT system and the results evaluated in terms of PITV, MDPD, and volume of brainstem receiving the prescribed dose. Our results suggest that both planning strategies can adequately cover the target and spare the organs at risk. The mMLC approach is superior in terms of mean PITV (1.42 vs 2.04) suggesting that the finer MLC resolution is advantageous, especially for smaller targets $(<3 \mathrm{cc})$. Subjectively, the IMRT plans produced smaller $25 \%$ and $50 \%$ isodose volumes. The IMRT plans resulted in greater organ at risk sparing (brainstem) at the cost of a less homogeneous distribution (mean MDPD 1.22 vs 1.11).

\section{The use of stereotactic radiotherapy with a micro-multileaf collimator for the treatment of choroidal melanoma}

Patrocinio H., Shenouda G., Deblois F., Parker W., Ruo R., Podgorsak E.,

McGill University Health Centre, Montreal, Quebec.

Choroidal melanoma is a disease generally confined to the eye. It presents technical treatment challenges for radiation therapy. Radioactive eye plaque is a standard treatment modality though it is difficult to place when the lesion lies near the optic nerve. Stereotactic radiotherapy using static conformal beams provides an elegant solution to treating these juxta-papillary lesions but eye immobilization becomes an important consideration. At our centre, an eye-positioning attachment to the stereotactic radiotherapy headring was built to provide both a light source that the patient could fix upon during treatment and a camera system that would allow for assessment of eye motion. The patient is CT-scanned with the eyepositioning device attached. The tumour is defined using manual MRI fusion and a PTV margin is added. A treatment plan is calculated using multiple conformal beams defined by a micromultileaf collimator. A dose of $60 \mathrm{~Gy}$ is currently prescribed in ten daily fractions to the periphery of the PTV. The patient is treated over a two-week period with the aid of the eye-positioning device. Our experience to date includes ten patients who received between $54 \mathrm{~Gy}$ and $60 \mathrm{~Gy}$ in ten fractions with this technique since April 2003. Preliminary clinical results suggest that the treatment is feasible and well tolerated with minor complications in two patients. It preserved or improved vision in nine patients and to date only one patient has required enucleation due to disease progression. Further follow-up is required to assess local control and complication rates.

\section{A critical analysis of conformity and homogeneity indices used in treatment plan evaluation for stereotactic radiosurgery}

Patrocinio H., Oliveira S., Souhami L., Podgorsak E., McGill University Health Centre, Montreal, Quebec.

The evaluation of treatment plans in stereotactic radiosurgery (SRS) requires an analysis of a large data set of isodose distributions and dose-volume histograms (DVH). Additional parameters are used 
to aid in plan evaluation: the PITV conformity index (ratio of volume within the planning isodose to the target volume), as well as the MDPD homogeneity index (ratio of the maximum dose to the prescription dose). Biological indices, such as tumor control probability (TCP) and normal tissue complication probability (NTCP), have been reported for this purpose but are not routinely used. In our study, PITV, MDPD, TCP and NTCP were used to evaluate plans calculated for a variety of target shapes and for two linac-based SRS techniques: the dynamic rotation technique and the micro-multileaf collimator conformal beam technique. Treatment plans were calculated for each target volume using both techniques and a variety of parameters (e.g. number of isocentres, beams, entry angles) and DVH analysis was used for determining PITV, MDPD, TCP and NTCP. The aim was to determine whether PITV and MDPD actually reflect the biological consequences of a given dose distribution as indicated by the TCP or NTCP and can lead to the choice of the "best" plan. A correlation between PITV and TCP or NTCP was found for the mMLC technique, while with the dynamic rotation technique, a similar correlation was not found particularly when using multiple isocentres. Further analysis suggested that dose variations inside the prescription isosurface results in TCP and NTCP variations even for similar conformity indices.

\section{Assessment of patient repositioning accuracy in stereotactic radiotherapy using mutual-information image fusion}

JL Robar, ${ }^{1}$ BG Clark, ${ }^{2}$ JW Schella ${ }^{1}$ and CS Kim ${ }^{1}$

${ }^{1}$ Nova Scotia Cancer Centre and Department of Radiation Oncology, Dalhousie University, Halifax, NS

${ }^{2}$ BC Cancer Agency-Vancouver Centre and Department of Physics and Astronomy, University of British Columbia, Vancouver, BC

This work describes a rapid and objective technique for determining repositioning error during the course of stereotactic radiation therapy using off-line CT imaging and automated mutualinformation image fusion within the treatment planning system (BrainSCAN, BrainLAB, AG). The method eliminates the variability associated with manual identification of anatomical landmarks by observers and allows quantification of translation and rotation of the cranium in three-dimensions. A phantom study was conducted to quantify the accuracy of the image fusion-based analysis itself. For CT voxel dimensions of $0.65 \times 0.65 \times 1.0 \mathrm{~mm}^{3}$, the method is shown to detect translations with an accuracy of 0.5 $\mathrm{mm}$ in the anterior-posterior and lateral dimensions and $0.8 \mathrm{~mm}$ in the superior-inferior dimension. Phantom rotations were detected to within 0.5 degrees of expected values. The analysis was applied to eight stereotactic radiotherapy patients at two independent clinics in Canada. Each patient was immobilized by the same mask system for cranial stereotactic radiotherapy and CT-imaged once per week over the five- to six-week course of treatment. Among all patients, the ranges of translation in the anterior-posterior, lateral and superiorinferior dimensions were $-0.91 \mathrm{~mm}$ to $0.77 \mathrm{~mm},-0.66 \mathrm{~mm}$ to 1.02 $\mathrm{mm}$ and $-2.24 \mathrm{~mm}$ to $3.47 \mathrm{~mm}$, respectively. Considering all patients and CT scans, the average repositioning errors were $0.16 \mathrm{~mm}, 0.04$ $\mathrm{mm}$ and $0.02 \mathrm{~mm}$ in the anterior-posterior, lateral and superiorinferior dimensions, respectively. The ranges of rotation about the superior-inferior, left-right and anterior-posterior axes were -2.84 to 2.62 degrees, -1.74 to 1.96 degrees and -1.78 to 1.42 degrees, respectively.

\section{Current radiosurgical approach to acoustic neuromas at McGill university: a preliminary report}

David Roberge M.D. ${ }^{1}$, Horacio Patrocinio M.S. ${ }^{2}$, Francois Deblois PhD. ${ }^{2}$, Luis Souhami M.D. ${ }^{1}$

${ }^{1}$ Department of Oncology, Division of Radiation Oncology, McGill University, Montreal, Quebec, Canada.

${ }^{2}$ Department of Medical Physics, McGill University, Montreal, Quebec, Canada

Following the use of other fractionation schemes, we adopted a new standardized approach to the treatment of acoustic neuromas in June 2003. Since then, 16 patients have been treated with fractionated stereotactic radiotherapy to a dose of $45 \mathrm{~Gy}$ in 20 daily fractions of $2.25 \mathrm{~Gy}$. These treatment courses are given using multiple (median 5) non-coplanar static beams delivered with the use of a micro-MLC tertiary collimator and non-invasive immobilization.

The patient's ages ranged from 15 to $63.4 / 16$ suffered from type II neurofibromatosis. Median maximal tumor diameter was $16 \mathrm{~mm}$ (7-31mm) with PTVs ranging from 1.34 to $28.47 \mathrm{cc}$. 11/16 patients had serviceable pre-treatment hearing (defined as speech recognition threshold greater than $50 \mathrm{db}$ or a speech discrimination percentage less than 50). The median prescription isodose was 93\% (85.7-95.7), median PITV 1.52 (1.25-1.7).

There were no severe acute toxicities. With a short median follow-up (six months), two patients have had dysfunction of cranial nerve VII (resolved in one case, of new onset in the other). One patient has suffered persistent vestibular toxicity. 4/9 evaluable patients with useful pre-radiation hearing now have now lost serviceable hearing. No patient has required surgery for tumor progression.

Fractionated stereotactic radiotherapy at a dose of $45 \mathrm{~Gy} / 20$ fractions has a favorable non-audiologic acute toxicity profile but, even with limited follow-up, results in significant hearing loss.

\section{Radiosurgery of the Spine and Cord: Where we want to go} Samuel Ryu, M.D.

Departments of Radiation Oncology and Neurosurgery, Henry Ford Hospital, Detroit, Michigan

Radiosurgery requires accurate targeting and immobilization of the target organ during irradiation. The difficulty of applying radiosurgery to the extra-cranial site is mainly due to organ motion associated with breathing and/or lack of immobilization techniques. Among the extracranial organs, the spinal cord/vertebrae are the organs with the least or no breathing-related organ movement. This makes the spine particularly suitable for stereotactic radiosurgery.

The spinal radiosurgery is performed by stereotactic body frame or by frameless image guided approach. Frameless stereotaxis is achieved by using metallic seed as reference guidance or by registering a reliable internal volume within the stereotactic range and image fusion. Using the available radiosurgery devices, clinical studies determined the precision of spinal radiosurgery within 1.5 $\mathrm{mm}$. We continued clinical efficacy study of spinal radiosurgery with dose escalation 10 Gy to 18 Gy single dose in 2 Gy increment for previously untreated single spine metastasis. Rapid pain control was achieved with median time to pain control/reduction 14 days following radiosurgery. Pain relief was durable. Radiologic tumor 
control was also seen in patients with epidural mass or soft tissue tumor component. Neurological improvement of motor and sensory function was achieved in a timely manner. The dose to the spinal cord was tolerable. Efforts were made to limit the cord dose under 10 Gy to the anterior $20 \%$ of the spinal cord. There was no detectable acute or subacute radiation toxicity noted clinically during the maximum followup time of 36 months. Image-guided spinal radiosurgery is accurate and safe, and achieves tumor control as well as significant clinical improvement of pain and neurologic function.

The available clinical results indicate clinical effectiveness of spinal radiosurgery for malignant and benign tumors of the spine or cord. Although progress has been made to use radiosurgery in the clinic, there are many more issues to improve and to explore the more sophisticated biological and clinical application. Basic research and clinical studies will be needed to further develop spinal radiosurgery as an important treatment option for various spinal disorders.

\section{The Development of Radiosurgery in Canada}

\section{Michael Schwart}

Pioneering Days: Radiosurgery began in Canada at McGill University, where the first patient was treated in 1986. Early development was characterized by innovation with the conception of the linear accelerator (linac) dynamic rotation technique by Podgorsak, and serviceable software written by Peters et al. They used the Olivier-Bertrand-Tipal (OBT) stereotactic frame with coregistration of several imaging modalities. The University of Toronto implemented dynamic rotation radiosurgery and treated their first patient in 1988. Quality assurance: One early theme in Toronto was accuracy and precision, and comparison with the "Gamma Knife" technique. The "Lucy" phantom was designed (Ramani et al.) and produced. Davey investigated the precision of target definition. Evaluating outcome: An algorithm for the prediction of AVM obliteration was developed (Schwartz et al.), allowing comparison of different centres and techniques. Szumacher et al. (Toronto) and Chung et al. (Vancouver) have published the outcome of fractionated stereotactic radiotherapy (SRT) for vestibular schwannomas.

New equipment: Various centres have responded in different ways to the need for new equipment for SRT. McGill has used a spinal injury halo, Toronto has modified an existing frame and Ottawa (Szanto et al.) has devised their own relocatable frame.

Wider dissemination of radiosurgery capacity: Calgary, Halifax, Winnipeg and Sherbrooke now all have radiosurgery; sophisticated linac systems in the two former and Gamma Knife units in the latter two centres.

Future development: Effort will shift from local hardware design to refinement of radiation technique, the pooling of experience, where in Canada we have a unique opportunity, and the evaluation of outcome.

\section{Liquid embolization material experimentally reduces the radiation dose delivered}

Daryl Scora, Yuri M. Andrade-Souza, Meera Ramani, David Beachey, May N. Tsao, Karel terBrugge, Michael L. Schwartz

Division of Neurosurgery and Department of Radiation Oncology, Sunnybrook and Women's College Health Sciences Centre; University of Toronto, Ontario, Canada

Objective: To test the hypothesis that the glue mixture (Embucrilate + Lipiodol) used for AVM embolization may decrease the dose delivered during radiosurgical treatment.

Method: A model was created using a block of solid water $(6 \times 5$ $\mathrm{x} 2 \mathrm{~cm}$ ). Twelve wells were drilled in the block to four different depths $(5 \mathrm{~mm}, 10 \mathrm{~mm}, 15 \mathrm{~mm}$ and $18 \mathrm{~mm})$ so that each row contained four wells with different depths and each column contained three wells with different concentrations of the glue mixture (Column 1: $80 \%$ contrast / 20\% glue; column 2: $50 \%$ contrast / 50\% glue and column 3: $20 \%$ contrast / $80 \%$ glue). The model was irradiated using a $5 \mathrm{MV}$ beam (LINAC system) and dose recorded with XV film at 7 $\mathrm{cm}$ depth, $10 \mathrm{~cm} \mathrm{SAD}$, with $15 \mathrm{~cm}$ backscatter.

Results: The reduction in dose varied with distance beyond the glue interface. For a distance of $0 \mathrm{~mm}, 2 \mathrm{~mm}$ and $5 \mathrm{~mm}$, the mean dose reduction was $13.65 \%(\mathrm{SD}=2.94), 6.87 \%(\mathrm{SD}=1.95)$ and $1.75 \%(\mathrm{SD}=1.14)$, respectively. There was also correlation with the Lipiodol concentration in the mixture. The maximum dose reductions for $80 \%, 50 \%$ and $20 \%$ Lipiodol concentrations are $16.1 \%(\mathrm{SD}=1.32), 14.85 \%(\mathrm{SD}=0.98)$ and $10 \%(\mathrm{SD}=1.21)$, respectively. There was no correlation between the glue depth and the dose delivered at the interface. Results of Monte Carlo (MCNP) modeling of this system (in progress) will be presented.

Conclusion: The hypothesis that the glue mixture used for embolization reduces the radiation dose delivered was experimentally confirmed.

\section{Regional Perfusion Changes in the Brain after Fractionated Stereotactic Radiotherapy}

D. Spencer ${ }^{*}$, C. Igna*, I. Kay ${ }^{*}$, A. Chan ${ }^{\S}$, Z. Kiss ${ }^{\ddagger}$, R. Frayne

Alberta Radiosurgery Center, Calgary, Alberta, Canada

* Department of Physics and Astronomy, University of Calgary

$\S$ Department of Oncology, University of Calgary

¥ Department of Neurosciences, University of Calgary

When irradiating the normal brain, one of the principal causes of complications is damage to the cerebral vasculature, particularly the micro-vasculature. This is of particular concern when treating diseases which are slow-growing or are not malignant. While there is some data relating loss of perfusion to radiation dose in other tissues such as lung, there is very little data for the brain. We are measuring relative regional Mean Transit Time (rrMTT) and relative regional Cerebral Blood Volume (rrCBV) before the beginning and after the completion of a course of radiation therapy. The patients had previously been treated with surgery, but had received no chemotherapy. Since the normal tissues of each patient receive a range of doses from zero to the prescribed dose, it is possible, in principle, to construct a complete dose-response curve from a single patient. However, limitations in the registration between data sets and signal/noise considerations make this not feasible. Still, a single 
patient can show the tendencies in perfusion changes, particularly at the high-dose end where the changes are largest.

We acquired data for two patients. We used a 3.0 T magnet at the Seaman Family MRI Centre at the Foothills Medical Centre in the form of perfusion weighted images using a single-shot echoplanar imaging sequence following the injection of a paramagnetic contrast agent (Gd-DTPA-Magnevist; Berlex, Wayne, NJ). These images have been processed to yield $\operatorname{rrCBV}$ and $\operatorname{rrMTT}$ hemodynamic measures.

Our preliminary results show that with a follow-up time of three months after receiving $5000 \mathrm{cGy} / 25$ fractions, in normal brain tissue the rrMTT is reduced by $3 \%$ and the $\mathrm{rCBV}$ is reduced by $12 \%$. It is expected that these changes will increase with longer term followup.

\section{Stereotactic Radiosurgical Activity in Canada: A Survey}

Brian D. Toyota, M.D., FRCSC

University of British Columbia

Although stereotactic radiosurgery (SRS) has been in use for decades, its place as primary treatment for some neurosurgical diseases is only now being verified. Canada has recently seen a flourish of SRS centres, including the addition of two Gamma knife units $^{\mathrm{TM}}$.

The individual numbers and years of experience of the Canadian experience is relatively small in comparison to other international sites. However Canada has a close-knit neurosciences community, each one functioning under similar heath care environments. As such there is an opportunity for Canadian centres to coalesce their experiences into a unified database. Canada-wide collaborative efforts can also be initiated for both retrospective and prospective SRS studies.

As a pre-requisite for such an endeavor it is felt that a base-line, descriptive landscape of the SRS activity in Canada is necessary.

An electronic-survey was distributed to ten Canadian sites that perform stereotactic radiosurgery- eight utilizing an modified linear accelerator and two Gamma Knife ${ }^{\mathrm{TM}}$ units.

The survey aimed to establish a description of the infra-structure, a list of names of the professionals involved, a summary of the processes used and a rough estimate of the volumes and treatment algorithms.

The results of this survey will be presented, and high-lights made of the potential for a Canadian radiosurgical database/consortium to make a significant impact on the international SRS literature.

\section{Fractionated stereotactic radiotherapy (SRT) for pituitary adenomas}

$D$ Voduc MD; M McKenzie MD; E Vollans MSc; BG Clark PhD; $R M a M D$

Radiation Oncology, BC Cancer Agency and Neurosurgery, Vancouver General Hospital, Vancouver, BC.

Background: Case series have reported the efficacy of single fraction radiosurgery (SRS) in the treatment of pituitary adenomas. Fractionated treatments can potentially reduce the risk of long term radiation side effects, while delivering a higher dose of radiation to the tumor. The objective of this study was to evaluate the radiological and biochemical pituitary tumor control rates following fractionated radiotherapy (SRT) at the BC Cancer Agency.

Materials and Methods: This study is a retrospective analysis of the first 37 patients with pituitary adenoma treated with SRT at the BCCA. SRT was delivered with a six MV linear accelerator using arcs or conformal beams. The prescribed dose was 50.4 Gy in 28 fractions, delivered five days per week. The outcomes recorded were radiological tumor control and biochemical control. The toxicity outcomes were hypopituitarism and visual loss.

Results: Thirty-six patients were treated for residual or recurrent disease after surgery, and one patient received SRT as first treatment. The median follow-up was 19.1 months. At the time of publication the radiological tumor control rate is $100 \%$. The two year actuarial rate of biochemical control is $30 \%$. Regarding toxicity, the two year actuarial rate of hypopituitarism is $20 \%$. No patients were found to have visual loss after SRT.

Conclusion: This study suggests that SRT is an effective and safe treatment for recurrent or residual pituitary adenoma.

\section{Radiosurgery for inoperable high-grade brain arteriovenous malformations and cavernous malformations: To treat or not?}

Wong JH, Kiss Z, Chan A, Parney I, Yan E, Craighead P

Alberta Radiosurgery Centre, Calgary, Alberta

The cure of high-grade brain arteriovenous malformations (AVMs) remains elusive despite advances in surgical and endovascular therapies, and their large lesional size has typically precluded successful obliteration with "traditional" radiosurgical approaches. Fractionated radiotherapy and staged radiosurgery techniques have been proposed and reported as a potential means of treating these challenging lesions. Similarly, radiosurgery has been used to treat inoperable cerebral cavernous malformations but controversy continues to dog the effectiveness and safety of this technique. We review the pertinent medical literature and propose a possible organized multi-centre protocol for the radiosurgical treatment of high-grade AVMs and cavernous malformations.

\section{Pediatric arteriovenous malformation: University of Toronto Experience with LINAC Radiosurgery}

Gelareh Zadeh, Yuri Souza, May Tsao, David Scora, Michael Schwartz

Sunnybrook and Women's Hospital, Toronto Regional Cancer Center, University of Toronto, Toronto, Canada

Introduction: Stereotactic radiosurgery has become an accepted treatment modality in the management of pediatric arteriovenous malformations (AVM). We present long-term outcomes for a series of pediatric AVM treated with LINAC based radiosurgery at the University of Toronto.

Methods: Between 1994 and 2004, out of the 244 cases of AVM treated with radiosurgery, 40 were under 18 years of age. A multidisciplinary team at the University of Toronto, composed of vascular neurosurgeons, interventional neuro-radiologist and radiation oncologist reviewed each individual case and decision regarding management option and modality was made in 
consultation with family. In eloquent brain a marginal dose of $15 \mathrm{~Gy}$ at the $67 \%$ isodose contour and in non-eloquent brain a marginal dose of 29 Gy at the $90 \%$ isodose contour was administered using a linear accelerator modified for radiosurgery. Application of the stereotactic head frame was performed under general anesthesia for patients less than 14 years of age. A concurrent intra-arterial digital angiography and dynamic CT scan with selective intra-arterial injection of contrast material was performed. We had complete follow-up for 36 months on 30 patients.

Results: The mean age of the patients was 12 and median age was 13 years. Majority of patients presented with hemorrhage (23 out of 37) followed by three patients with seizures, six with headaches and one incidental presentation. The percentage of patients presenting with hemorrhage was significantly higher than our adult population. Complete angiographic follow-up was available on 30 patients $67 \%$ showed complete obliteration of the AVMs. One patient had immediate post radiosurgery seizures with no long-term effects and four patients developed long-term complications: one hand tremor due to a small infarct, two hemiparesis not limiting function and one hemianopsia.

Conclusions: Our LINAC based treatment of pediatric AVMs provides a safe and efficacious management option, with comparable results to other series using either Gamma Knife or LINAC radiosurgery. Our obliteration rate and low complications supports a pivotal role for LINAC radiosurgery in treatment of pediatric AVMs.

\section{ACKNOWLEDGMENTS}

Many thanks go to the local organizing committee, Drs. Alex Chan, Peter Craighead, Elizabeth Yan (Radiation Oncology, Calgary), Drs. David Spencer, Chris Newcomb, and Karen Breitman (Medical Physics, Calgary). The members of the abstract review committee, Drs. Chris Newcomb, Michael West (Winnipeg), Shawn Malone (Ottawa) and Ian Fleetwood (Halifax), are commended for their efforts in fostering this meeting's impressive standards. We extend our gratitude to Ms. Joan Sweeney from the University of Calgary Continuing Medical Education office for her outstanding logistical and administrative support.

\section{Disclosure}

Unrestricted educational grant support for this meeting was received from BrainLAB $\mathrm{GmbH}$, TomoTherapy Incorporated, Accuray Incorporated, and Varian Medical Systems.

Reprint requests to: Zelma Kiss, Room 182A, Heritage Medical Research Building, 3330 Hospital Drive NW, Calgary, Alberta, T2N 4N1, FAX: 403-283-8731 Email: zkiss@ucalgary.ca 